The Astrophysical Journal, 614:122-134, 2004 October 10

(C) 2004. The American Astronomical Society. All rights reserved. Printed in U.S.A.

\title{
NEAR-INFRARED $K$-BAND SPECTROSCOPIC INVESTIGATION OF SEYFERT 2 NUCLEI IN THE CfA AND 12 MICRON SAMPLES
}

\author{
MASATOSHI IMANishi ${ }^{1}$ \\ National Astronomical Observatory, 2-21-1, Osawa, Mitaka, Tokyo 181-8588, Japan; imanishi@optik.mtk.nao.ac.jp \\ AND \\ Almudena Alonso-Herrero \\ Departamento de Astrofísica Molecular e Infrarroja, IEM, Consejo Superior de Investigaciones Científicas, \\ Serrano 113b, 28006 Madrid, Spain; aalonso@damir.iem.csic.es \\ Received 2004 April 12; accepted 2004 June 17
}

\begin{abstract}
We present near-infrared $K$-band slit spectra of the nuclei of 25 Seyfert 2 galaxies in the CfA and $12 \mu$ m samples. The strength of the $\mathrm{CO}$ absorption features at 2.3-2.4 $\mu \mathrm{m}$ produced by stars is measured in terms of a spectroscopic CO index. A clear anticorrelation between the observed $\mathrm{CO}$ index and the nuclear $K-L$ color is present, suggesting that a featureless hot dust continuum heated by an active galactic nucleus (AGN) contributes significantly to the observed $K$-band fluxes in the nuclei of Seyfert 2 galaxies. After correction for this AGN contribution, we estimate nuclear stellar $K$-band luminosities for all sources and $\mathrm{CO}$ indices for sources with modestly large observed CO indices. The corrected CO indices for $10(=40 \%)$ Seyfert 2 nuclei are found to be as high as those observed in star-forming or elliptical (=spheroidal) galaxies. We combine the $K$-band data with measurements of the $L$-band $3.3 \mu \mathrm{m}$ polycyclic aromatic hydrocarbon (PAH) emission feature, another powerful indicator for star formation, and find that the $3.3 \mu \mathrm{m}$ PAH to $K$-band stellar luminosity ratios are substantially smaller than those of starburst galaxies. Our results suggest that the $3.3 \mu \mathrm{m}$ PAH emission originates in the putative nuclear starbursts in the dusty tori surrounding the AGNs, because of its high surface brightness, whereas the $K$-band $\mathrm{CO}$ absorption features detected at the nuclei are dominated by old bulge (=spheroid) stars and thus may not be a powerful indicator for the nuclear starbursts. We see no clear difference in the strength of the $\mathrm{CO}$ absorption and PAH emission features between the CfA and $12 \mu \mathrm{m}$ Seyfert 2 galaxies.
\end{abstract}

Subject headings: galaxies: active — galaxies: nuclei — galaxies: Seyfert — infrared: galaxies

\section{INTRODUCTION}

According to the current unification paradigm of Seyfert galaxies - the most numerous class of active galactic nuclei (AGNs) in the local universe - the two types of Seyfert galaxies, type 1 (which show broad optical emission lines) and type 2 (which do not), are both powered by a central massaccreting supermassive black hole. The different emission properties are explained by the presence of a torus-shaped structure of molecular gas and dust surrounding the central supermassive black hole (the so-called dusty torus) and the different viewing angles toward the torus (Antonucci 1993). Since the dusty torus is rich in molecular gas, it is a natural site for starbursts to occur (Fabian et al. 1998). Such a nuclear starburst in the torus could play an important role for AGN physics (Wada \& Norman 2002), so that its observational understanding is very important.

Attempts to put observational constraints on the nuclear starbursts in the torus in Seyfert 2 galaxies have been made extensively, from UV to optical (e.g., Gonzalez Delgado et al. 1998), through infrared (e.g., Oliva et al. 1999; Ivanov et al. 2000; Imanishi 2002, 2003), to millimeter wavelength ranges (Kohno et al. 2002). Among these methods, infrared diag-

\footnotetext{
${ }^{1}$ Visiting Astronomer at the Infrared Telescope Facility, which is operated by the University of Hawaii under cooperative agreement NCC 5-538 with the National Aeronautics and Space Administration, Office of Space Science, Planetary Astronomy Program.
}

nostics have two major advantages. First, since nuclear starbursts in the torus are expected to have some amount of dust extinction, infrared observations are better suited to investigate their properties than those in the UV or optical, because of the reduced effects of dust extinction in the infrared. Second, compared to millimeter observations, a systematic study of the nuclear starbursts in a statistically meaningful number of Seyfert 2 galaxies is possible with a reasonable amount of telescope time. Imanishi (2003) obtained infrared $L$-band (2.8$4.1 \mu \mathrm{m}$ ) slit spectra of 32 Seyfert 2 galaxies in the CfA (Huchra $\&$ Burg 1992) and $12 \mu \mathrm{m}$ (Rush et al. 1993) samples. Utilizing the polycyclic aromatic hydrocarbon (PAH) emission feature at $3.3 \mu \mathrm{m}$ as an excellent tool to disentangle star formation from AGN activity (Imanishi 2002), the author discussed the properties of nuclear starbursts in Seyfert 2 galaxies in a quantitative manner.

Nuclear starbursts in Seyfert 2 galaxies can also be investigated through infrared $K$-band $(2.0-2.5 \mu \mathrm{m})$ spectroscopy. Since the CO absorption features at $\lambda_{\text {rest }}=2.3-2.4 \mu \mathrm{m}$ in the rest frame are produced by stars, and not by a pure AGN, these features can be a good tool to distinguish between stars and an AGN (Oliva et al. 1999; Ivanov et al. 2000). Among the 32 Seyfert 2 galaxies studied by Imanishi (2003), 25 sources were observed with SpeX at the IRTF $3 \mathrm{~m}$ telescope (Rayner et al. 2003) (see Table 2 of Imanishi 2003). Since Spex enables us to obtain $K$ - and $L$-band spectra simultaneously, the stellar emission in Seyfert 2 nuclei can be investigated also from the $K$-band $\mathrm{CO}$ absorption features, independently from the $L$-band PAH diagnostic. In this paper, we present $K$-band 
TABLE 1

Details of the Observed Seyfert 2 Galaxies

\begin{tabular}{|c|c|c|c|c|c|c|c|c|c|}
\hline \multirow[b]{2}{*}{$\begin{array}{c}\text { ОвJест } \\
\text { (1) }\end{array}$} & \multirow[b]{2}{*}{$\begin{array}{c}\text { REDSHIFT } \\
\text { (2) }\end{array}$} & \multirow[b]{2}{*}{$\begin{array}{c}\text { Physical Scale } \\
(\mathrm{kpc}) \\
(3)\end{array}$} & \multirow[b]{2}{*}{$\begin{array}{l}\text { DATE } \\
\text { (UT) } \\
(4)\end{array}$} & \multirow[b]{2}{*}{$\begin{array}{l}\text { INTEGRATION } \\
\text { (minutes) } \\
\text { (5) }\end{array}$} & \multicolumn{4}{|c|}{ STANDARd STAR } & \multirow[b]{2}{*}{$\begin{array}{c}\text { Comments } \\
(10)\end{array}$} \\
\hline & & & & & $\begin{array}{c}\text { Name } \\
(6)\end{array}$ & $\begin{array}{c}K \text { Magnitude } \\
\text { (7) }\end{array}$ & $\begin{array}{l}\text { Type } \\
(8)\end{array}$ & $\begin{array}{r}T_{\text {eff }} \\
(\mathrm{K}) \\
(9)\end{array}$ & \\
\hline Mrk $993 \ldots .$. & 0.015 & 0.46 & 2002 Aug 27, 29 & 100 & HR 410 & 5.0 & F7 V & 6240 & CfA \\
\hline Mrk 573 ............................ & 0.017 & 0.52 & 2002 Aug 28 & 30 & HR 650 & 4.2 & $\mathrm{~F} 8 \mathrm{~V}$ & 6000 & CfA \\
\hline 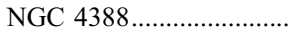 & 0.008 & 0.25 & 2003 Mar 18 & 30 & HR 4708 & 5.0 & F8 V & 6000 & CfA, $12 \mu \mathrm{m}$ \\
\hline 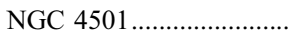 & 0.008 & 0.25 & 2003 Mar 18 & 40 & HR 4708 & 5.0 & F8 V & 6000 & $\mathrm{CfA}^{\mathrm{a}}{ }^{\mathrm{a}} 12 \mu \mathrm{m}$ \\
\hline 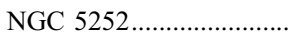 & 0.023 & 0.69 & 2003 Mar 19 & 40 & HR 5011 & 3.8 & G0 V & 5930 & $\mathrm{CfA}$ \\
\hline NGC 5347....................... & 0.008 & 0.25 & 2003 Mar 18 & 40 & HR 5346 & 4.9 & F8 V & 6000 & CfA, $12 \mu \mathrm{m}$ \\
\hline 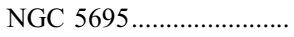 & 0.014 & 0.43 & 2003 Mar 20 & 60 & HR 5630 & 5.0 & F8 V & 6000 & CfA \\
\hline NGC 5929........................ & 0.008 & 0.25 & 2002 Aug 29 & 40 & HR 5728 & 4.6 & G3 V & 5800 & CfA, $12 \mu \mathrm{m}$ \\
\hline 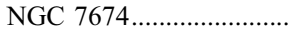 & 0.029 & 0.87 & 2002 Aug 27 & 20 & HR 8653 & 4.7 & G8 IV & 5400 & $\mathrm{CfA}, 12 \mu \mathrm{m}$ \\
\hline 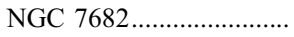 & 0.017 & 0.52 & 2002 Aug 27, 29 & 160 & HR 8969 & 2.8 & F7 V & 6240 & CfA \\
\hline 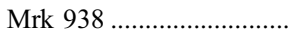 & 0.019 & 0.58 & 2002 Aug 28 & 30 & HR $8917^{b}$ & 5.0 & G0 V & 5930 & $12 \mu \mathrm{m}$ \\
\hline NGC $262($ Mrk 348)....... & 0.015 & 0.46 & 2002 Aug 28 & 30 & HR 410 & 5.0 & F7 V & 6240 & $12 \mu \mathrm{m}$ \\
\hline 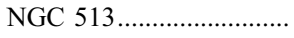 & 0.020 & 0.61 & 2002 Aug 28 & 30 & HR 410 & 5.0 & F7 V & 6240 & $12 \mu \mathrm{m}$ \\
\hline F01475-0740 .................. & 0.017 & 0.52 & 2002 Aug 27 & 30 & HR 466 & 4.9 & F7 V & 6240 & $12 \mu \mathrm{m}$ \\
\hline 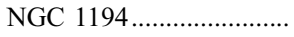 & 0.013 & 0.40 & 2002 Aug 28 & 30 & HR 996 & 3.3 & G5 V & 5700 & $12 \mu \mathrm{m}$ \\
\hline 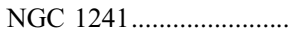 & 0.014 & 0.43 & 2002 Aug 29 & 50 & HR 784 & 4.6 & F6 V & 6400 & $12 \mu \mathrm{m}$ \\
\hline NGC 1320 (Mrk 607)..... & 0.010 & 0.31 & 2002 Aug 29 & 30 & HR 784 & 4.6 & F6 V & 6400 & $12 \mu \mathrm{m}$ \\
\hline F04385-0828 …............ & 0.015 & 0.46 & 2002 Aug 29 & 30 & HR 1536 & 4.4 & F8 V & 6000 & $12 \mu \mathrm{m}$ \\
\hline 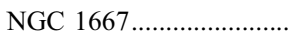 & 0.015 & 0.46 & 2003 Mar 20 & 60 & HR 1536 & 4.4 & F8 V & 6000 & $12 \mu \mathrm{m}$ \\
\hline 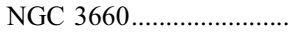 & 0.012 & 0.37 & 2003 Mar 20 & 90 & HR 4529 & 4.9 & F7 V & 6240 & $12 \mu \mathrm{m}$ \\
\hline 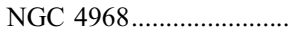 & 0.010 & 0.31 & 2003 Mar 20 & 30 & HR 4935 & 4.3 & F7 V & 6240 & $12 \mu \mathrm{m}$ \\
\hline MCG $-3-34-64 \ldots \ldots \ldots \ldots . .$. & 0.017 & 0.52 & 2003 Mar 19 & 40 & HR 4995 & 3.7 & G6 V & 5620 & $12 \mu \mathrm{m}$ \\
\hline MCG $-2-40-4 \ldots \ldots \ldots \ldots \ldots$ & 0.024 & 0.72 & 2003 Mar 19 & 40 & HR 5779 & 5.2 & F7 V & 6240 & $12 \mu \mathrm{m}$ \\
\hline F15480-0344 ................ & 0.030 & 0.90 & 2002 Aug 29 & 30 & HR 5779 & 5.2 & F7 V & 6240 & $12 \mu \mathrm{m}$ \\
\hline MCG - 3-58-7 .............. & 0.032 & 0.96 & 2002 Aug 28 & 20 & HR 8457 & 4.9 & F6 V & 6400 & $12 \mu \mathrm{m}$ \\
\hline
\end{tabular}

Notes.-Col. (1): object; col. (2): redshift; col. (3): physical scale of the employed slit width of 1".6; col. (4): observing date in UT; col. (5): net on-source

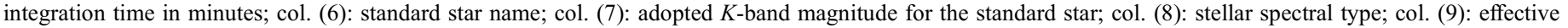
temperature in K; col. (10): CfA (Huchra \& Burg 1992) or $12 \mu \mathrm{m}$ (Rush et al. 1993) Seyfert 2 galaxies.

${ }^{a}$ NGC 4501 is classified as a LINER in the CfA sample (Huchra \& Burg 1992).

b Flux calibration was made using HR 8457.

spectroscopic results and combine them with the $L$-band results to provide a better understanding of Seyfert 2 nuclei; $H_{0}=75 \mathrm{~km} \mathrm{~s}^{-1} \mathrm{Mpc}^{-1}, \Omega_{M}=0.3$, and $\Omega_{\Lambda}=0.7$ are adopted throughout this paper.

\section{TARGETS, OBSERVATIONS, AND DATA ANALYSIS}

Detailed information and the observing log of the 25 Seyfert 2 galaxies are summarized in Table 1. The original sample selection criteria and observing details are found in Imanishi (2003). In short, Seyfert 2 galaxies in the CfA and $12 \mu \mathrm{m}$ samples at $z=0.008-0.035$ and at declination greater than $-35^{\circ}$ were selected. The CfA and $12 \mu \mathrm{m}$ Seyfert 2 galaxies were selected on the basis of their optical magnitudes (Huchra \& Burg 1992) and IRAS $12 \mu \mathrm{m}$ fluxes (Rush et al. 1993), respectively. The observations were made with SpeX at the IRTF $3 \mathrm{~m}$ telescope, using the 1.9-4.2 $\mu \mathrm{m}$ cross-dispersed mode with a 1 ." 6 wide slit (Rayner et al. 2003), which provides a spectral resolution of $R \sim 500$ in the $K$ band. The 1"6 aperture used corresponds to physical scales of between $250 \mathrm{pc}$ $(z=0.008)$ and $1 \mathrm{kpc}(z=0.035)$, so our spectra are suitable to investigate the nuclear starbursts rather than ring-shaped circumnuclear starbursts that are typically found at distances of $\sim 1 \mathrm{kpc}$ from the nuclei (Le Floc'h et al. 2001). The sky was clear, and the seeing at $K$ was $0.6-0$ ".9 (FWHM) throughout the observations.
The data reduction of the $K$-band spectra was made using IRAF $^{2}$ in the same way as the $L$-band data, as described by Imanishi (2003). The Seyfert 2 and standard star spectra were extracted by integrating signals over 1". $8-3$ ". 0 along the slit, depending on the actual signal profiles. The $K$-band magnitudes of the standard stars in Table 1 were estimated from their $V$-band $(0.6 \mu \mathrm{m})$ magnitudes, adopting $V-K$ colors appropriate to the stellar types of individual standard stars (Tokunaga 2000). The Seyfert 2 spectra were divided by those of the corresponding standard stars and then multiplied by the spectra of blackbodies with temperatures corresponding to those of the individual standard stars (Table 1). Appropriate spectral binning was employed, particularly for faint sources. The intrinsic $\mathrm{CO}$ absorption of the adopted standard stars (A-, F-, and G-type main-sequence stars) is too weak (Arnaud et al. 1989) to affect our main conclusions about the $\mathrm{CO}$ absorption strength of the Seyfert 2 nuclei.

\section{RESULTS}

Figure 1 shows the flux-calibrated $K$-band slit spectra of the 25 Seyfert 2 galaxies in our sample. Some sources showed a

${ }^{2}$ IRAF is distributed by the National Optical Astronomy Observatory, which is operated by the Association of Universities for Research in Astronomy (AURA), Inc., under cooperative agreement with the National Science Foundation. 

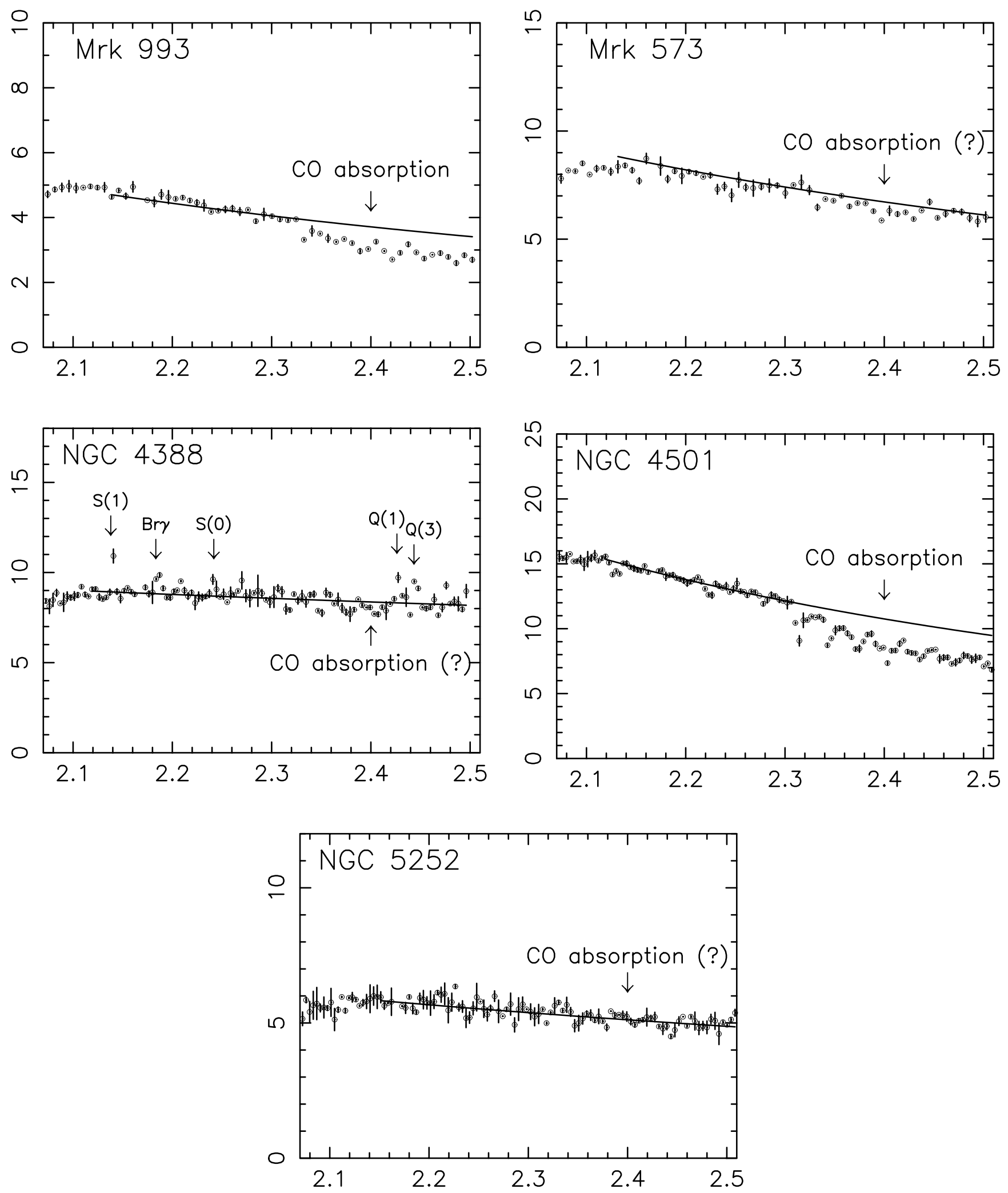

FIG. 1.-Infrared 2.07-2.5 $\mu \mathrm{m}$ spectra of the 25 Seyfert 2 nuclei. The abscissa and ordinate represent the observed wavelength in $\mu \mathrm{m}$ and flux $F_{\lambda}$ in $10^{-15} \mathrm{~W} \mathrm{~m}^{-2}$ $\mu \mathrm{m}^{-1}$, respectively. The solid lines represent adopted continuum levels, with respect to which the CO indices are measured. The arrows indicate the CO absorption features. When an observed CO index is greater (smaller) than 0.1, the term "CO absorption" ["CO absorption (?)"] is used. Some strong emission lines are indicated: $\mathrm{H}_{2} 1-0 S(1)$ emission line at $\lambda_{\text {rest }}=2.122 \mu \mathrm{m}$ [denoted as $\left.S(1)\right], \mathrm{Br} \gamma$ at $\lambda_{\text {rest }}=2.166 \mu \mathrm{m}(\mathrm{Br} \gamma), \mathrm{H}_{2} 1-0 S(0)$ at $\lambda_{\text {rest }}=2.223 \mu \mathrm{m}[S(0)], \mathrm{H}_{2} 1-0 Q(1)$ at $\lambda_{\text {rest }}=2.407 \mu \mathrm{m}[Q(1)]$, and $\mathrm{H}_{2} 1-0 Q(3)$ at $\lambda_{\text {rest }}=2.424 \mu \mathrm{m}[Q(3)]$. 

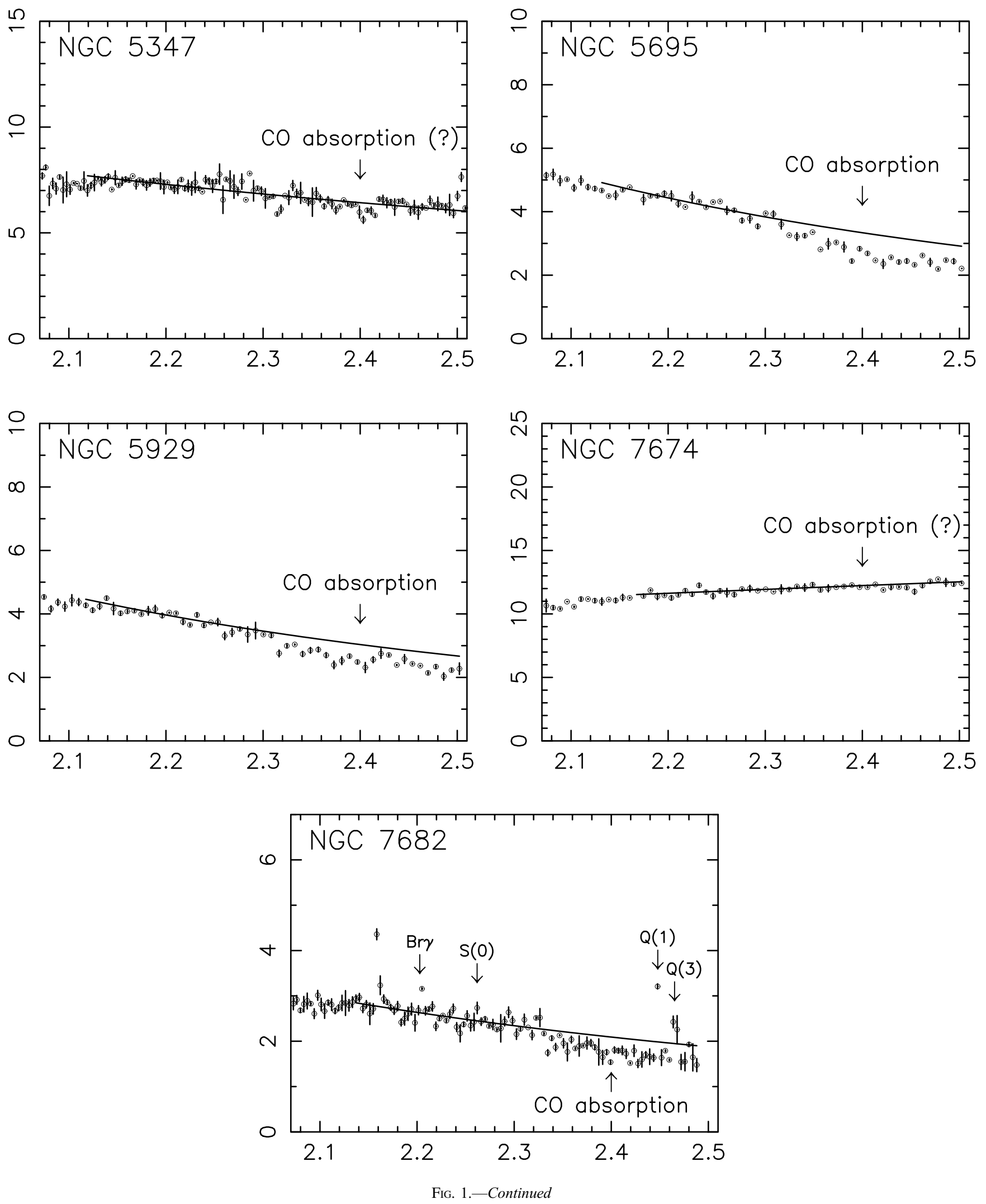

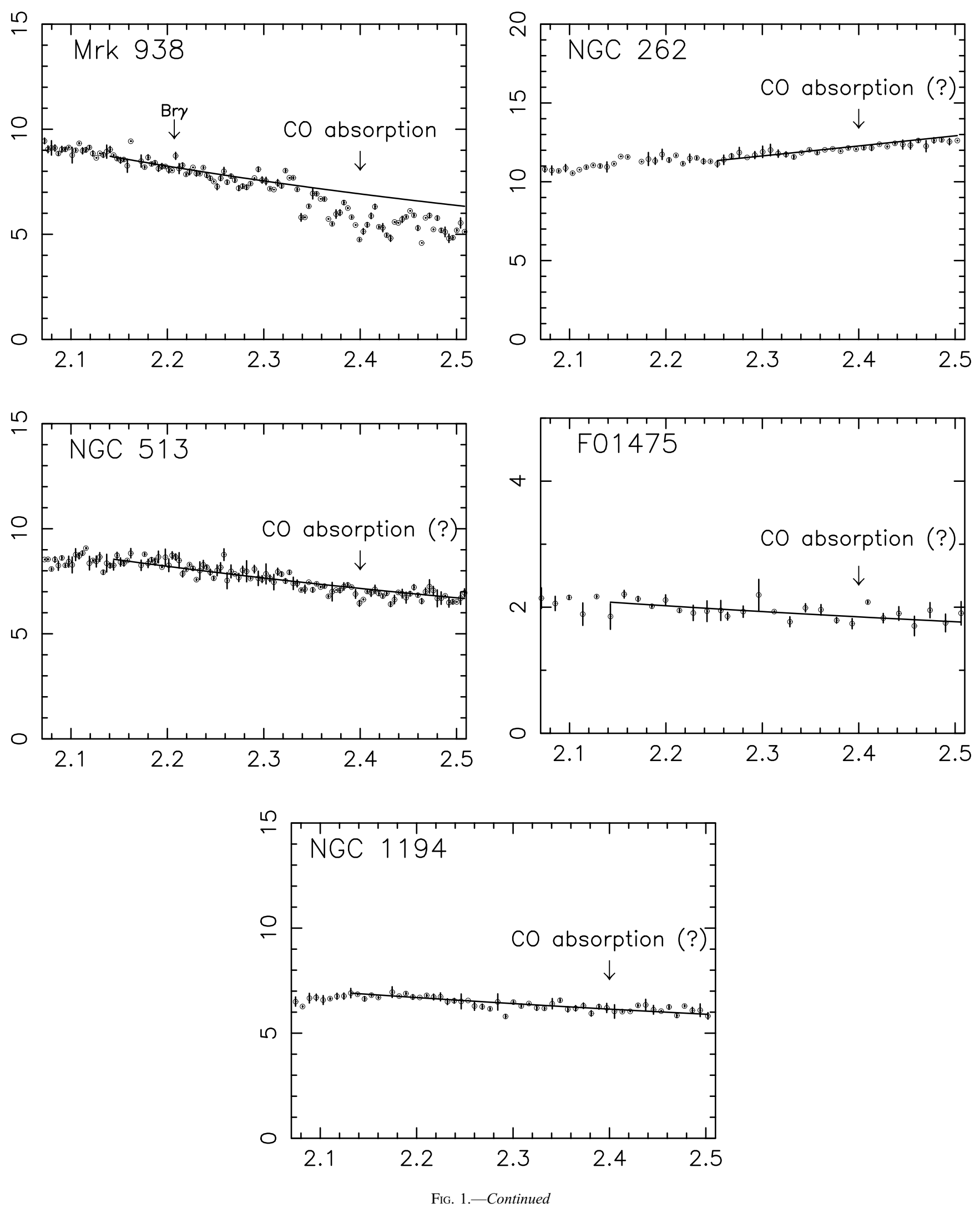

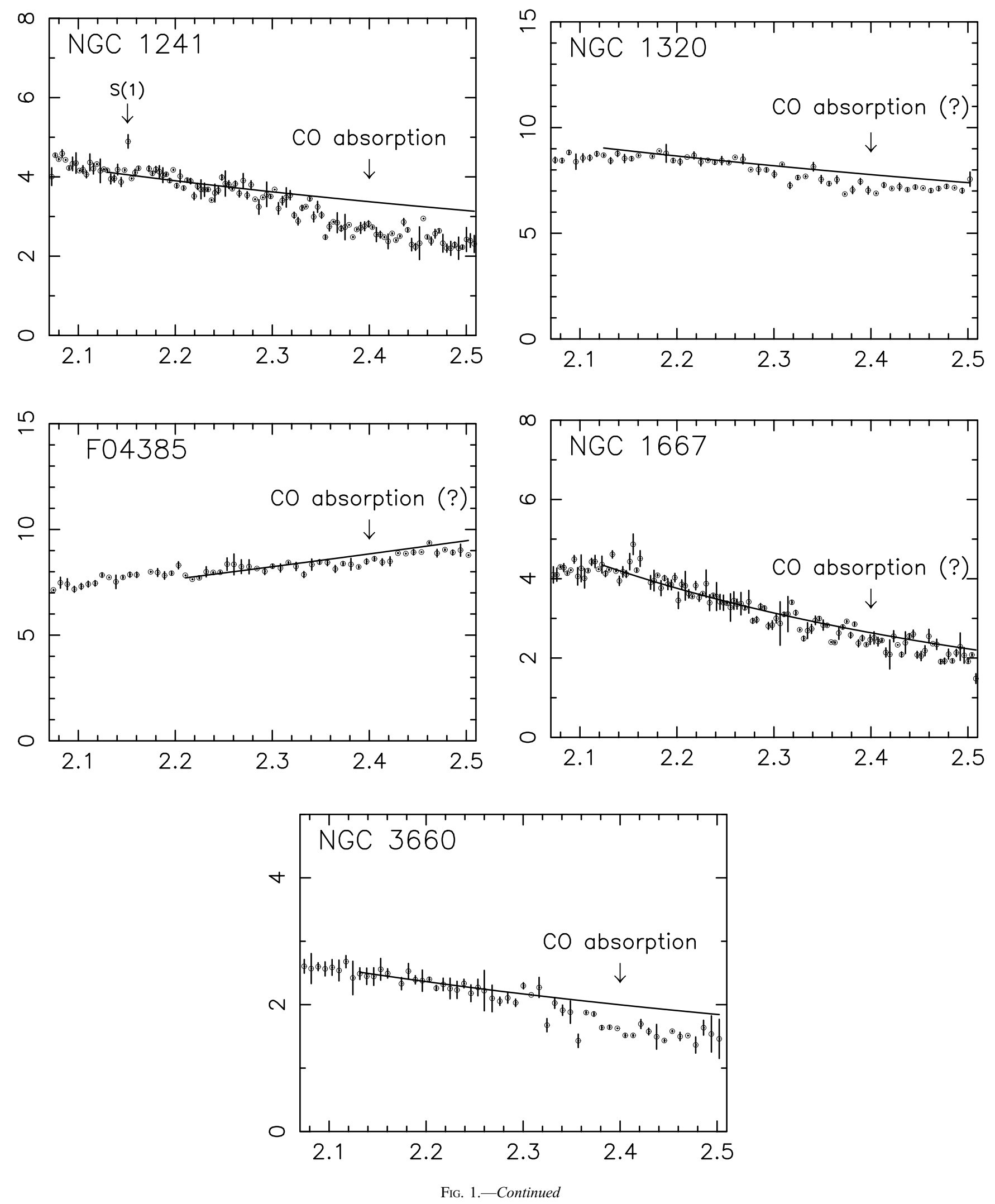

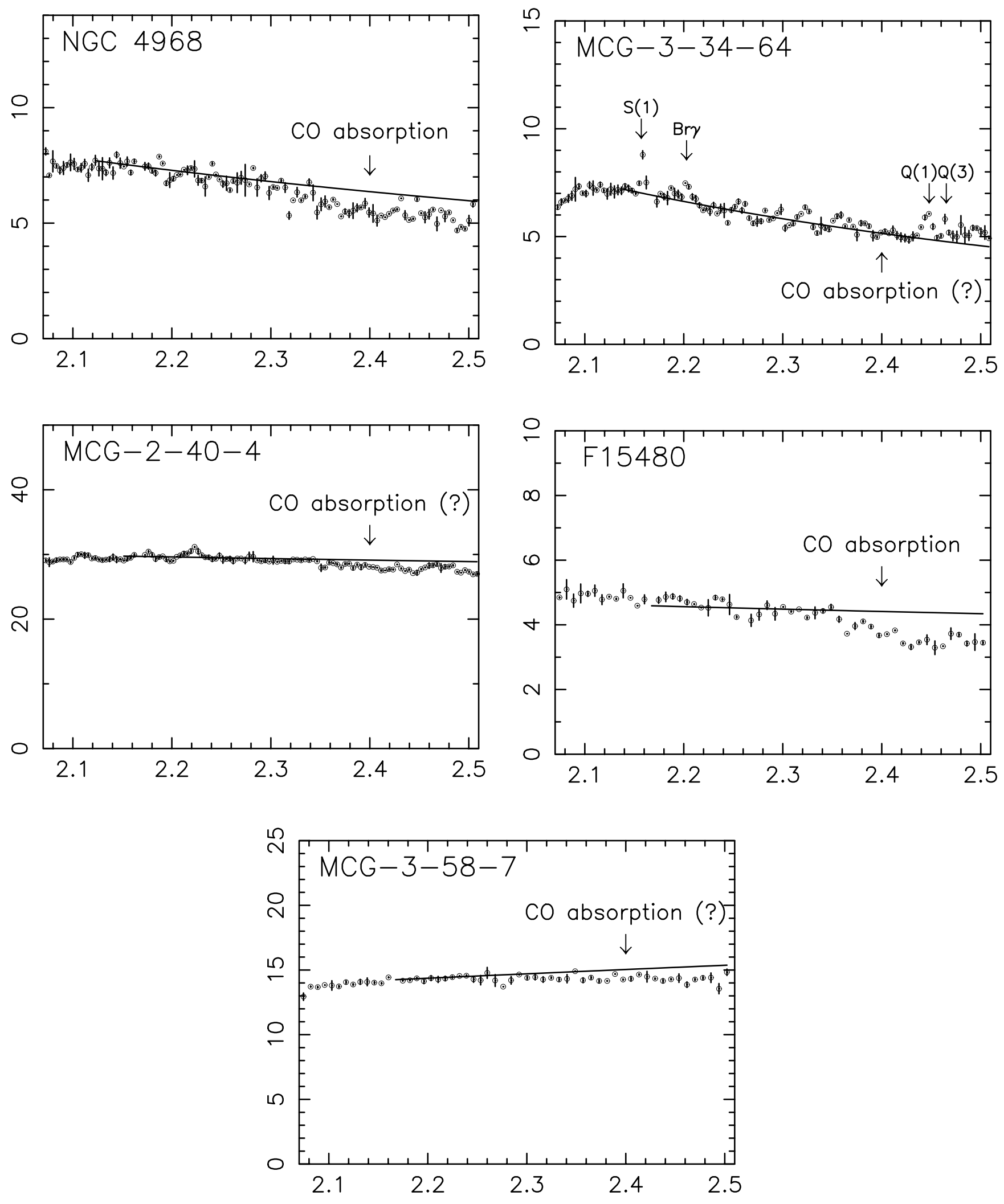

Fig. 1.-Continued 
larger scatter in the spectra over the $\lambda_{\mathrm{obs}}=2.00-2.02 \mu \mathrm{m}$ and 2.05-2.07 $\mu \mathrm{m}$ range in the observed frame than their neighboring wavelengths, owing to the presence of the Earth's atmospheric absorption features. Since these data points may have bad effects on the continuum determination, with which to estimate the strength of the $\mathrm{CO}$ absorption features, we only used spectra at $\lambda_{\text {obs }}=2.07-2.5 \mu \mathrm{m}$. For some of the Seyfert 2 galaxies in our sample, spectra with a smaller wavelength coverage, in the close vicinity of the $\operatorname{Br} \gamma$ emission line at $\lambda_{\text {rest }}=$ $2.166 \mu \mathrm{m}$, are available (Veilleux et al. 1997; Rhee \& Larkin 2000). For all the CfA Seyfert 2 galaxies in our sample except NGC 4501 and NGC 5347, Ivanov et al. (2000) presented moderate-resolution $(R \sim 700)$ spectra at $\lambda_{\text {rest }}=2.0-2.4 \mu \mathrm{m}$ and/or higher resolution $(R>1000)$ spectra in narrower wavelength ranges near the $\mathrm{CO}$ absorption features. However, for the majority of the 25 Seyfert 2 galaxies, our spectra in Figure 1 are the first moderate-resolution spectra with an almost complete $K$-band coverage. Thus, a systematic and consistent investigation of the $\mathrm{CO}$ absorption features in a statistically larger number of Seyfert 2 galaxies is possible.

To investigate the presence of nuclear starbursts in Seyfert 2 galaxies using the strength of the $\mathrm{CO}$ absorption features, we basically follow the method proposed by Doyon et al. (1994), who defined a spectroscopic $\mathrm{CO}$ index as

$$
\mathrm{CO}_{\text {spec }} \equiv-2.5 \log \left\langle R_{2.36}\right\rangle
$$

where $\left\langle R_{2.36}\right\rangle$ is the average of actual signals at $\lambda_{\text {rest }}=2.31-$ $2.40 \mu \mathrm{m}$ divided by a power-law continuum $\left(F_{\lambda}=\alpha \times \lambda^{\beta}\right)$ extrapolated from shorter wavelengths. When the $\mathrm{CO}$ absorption is present, $\left\langle R_{2.36}\right\rangle$ should be less than unity, and thus the $\mathrm{CO}_{\text {spec }}$ should show a positive value. This $\mathrm{CO}$ index is insensitive to spectral resolution, because an average value within the wavelengths that show CO absorption is used. Thus, this index can be directly compared among independent spectra observed with slightly different resolutions.

To determine the continuum level, Doyon et al. (1994) used data points at $\lambda_{\text {rest }}=2.0-2.29 \mu \mathrm{m}$, excluding clear emission lines, and extrapolated it to $\lambda_{\text {rest }}>2.31 \mu \mathrm{m}$. In our sample, the redshifts are in the range $z=0.008-0.035$ and only data points at wavelengths $\lambda_{\text {obs }}=2.07-2.5 \mu \mathrm{m}$ are available. Thus, data points at wavelengths as short as $\lambda_{\text {rest }}=2.0 \mu \mathrm{m}$ are not fully covered. We thus used data points at $\lambda_{\text {rest }}=2.1-2.29 \mu \mathrm{m}$ to determine the continuum level and extrapolated it to longer wavelengths. Following Doyon et al. (1994), a power-law shape was assumed for the continuum and clear emission lines, such as $\mathrm{H}_{2} 1-0 S(1)\left(\lambda_{\text {rest }}=2.122 \mu \mathrm{m}\right)$ or $\operatorname{Br} \gamma\left(\lambda_{\text {rest }}=2.166 \mu \mathrm{m}\right)$, were excluded. Regarding the wavelength range that should contain the $\mathrm{CO}$ absorption features, our spectra of $\lambda_{\mathrm{obs}}=$ 2.07-2.5 $\mu \mathrm{m}$ covers $\lambda_{\text {rest }}=2.31-2.4 \mu \mathrm{m}$ for all the observed Seyfert 2 galaxies at $z=0.008-0.035$, so that the same wavelength range of $\lambda_{\text {rest }}=2.31-2.4 \mu \mathrm{m}$ as employed by Doyon et al. (1994) was adopted to measure the CO absorption strength. Although the wavelength range used for the continuum determination is slightly different, our spectroscopic $\mathrm{CO}$ index is essentially the same as that defined by Doyon et al. (1994).

Whenever possible, we tried to use this method to determine the continuum level in a consistent way. However, in the case of NGC 262 and F04385-0828, the extrapolated continuum determined in this way provided a negative value for $\mathrm{CO}_{\text {spec, }}$, possibly because of the curvature of the continuum emission at $\lambda_{\text {rest }}=2.1-2.29 \mu \mathrm{m}$. In these two sources, we used only data at $\lambda_{\text {rest }}=2.22-2.29 \mu \mathrm{m}$ and $2.18-2.29 \mu \mathrm{m}$, respectively, which provided more reasonable extrapolated continuum levels at $\lambda_{\text {rest }}=2.31-2.40 \mu \mathrm{m}$. The adopted continuum levels are plotted as solid lines in Figure 1.

The values and histogram of the observed spectroscopic $\mathrm{CO}$ indices $\left(\mathrm{CO}_{\text {spec-obs }}\right)$ are summarized in column (2) of Table 2 and Figure $2 a$, respectively. The largest uncertainty of the $\mathrm{CO}_{\text {spec-obs }}$ values comes from the ambiguity of the continuum determination, which is difficult to assess quantitatively. For some Seyfert 2 galaxies, we slightly changed the wavelength range used for the continuum determination, as well as the removed data points that may be contaminated by emission lines. The $\mathrm{CO}_{\text {spec-obs value differs by a factor of as high as }}$ 0.03 , which can be taken as a possible maximum uncertainty for $\mathrm{CO}_{\text {spec-obs. }}$

For the majority of the CfA Seyfert 2 galaxies, Ivanov et al. (2000) estimated spectroscopic CO indices on the basis of independent data. They used a very narrow wavelength range of $\lambda_{\text {rest }}=2.285 \pm 0.005 \mu \mathrm{m}$ and $2.298 \pm 0.005 \mu \mathrm{m}$ for the continuum and the data points that include $\mathrm{CO}$ absorption features, respectively. If this method were to be applied to our lower resolution spectra, the resulting uncertainty for $\mathrm{CO}_{\text {spec-obs }}$ would be large, because only one or two data points would be included in each of these wavelength ranges. In Table 2 we compare the observed spectroscopic $\mathrm{CO}$ indices based on our wider wavelength coverage spectra with the indices derived by Ivanov et al. (2000). For some sources (NGC 5929, NGC 7674, and NGC 7682), both CO index measurements are in good agreement, but there is some discrepancy for other sources, possibly because of the different method to derive $\mathrm{CO}_{\text {spec-obs }}$ and/or different aperture size.

In some objects, narrow ( $<500 \mathrm{~km} \mathrm{~s}^{-1}$ in FWHM) emission lines are discernible in the spectra. In Figure 1, we indicate the identification of some clear emission lines. In general, their equivalent widths are smaller than in starburst galaxies (Goldader et al. 1997b; Vanzi et al. 1998; Coziol et al. 2001). To quantitatively estimate these weak and narrow emission line fluxes, by tracing the line profiles, spectra with $R>1000$ are usually required. No quantitative estimates for the line fluxes are made on the basis of our lower resolution $(R \sim 500)$ spectra.

\section{DISCUSSION}

\subsection{CO Absorption Strength}

Unlike pure star-forming galaxies, a featureless continuum from hot dust heated by an AGN can contribute significantly to the observed $K$-band fluxes in Seyfert 2 galaxies. This contribution reduces the depth of the $\mathrm{CO}$ absorption feature; the degree of this reduction can vary, depending on the amount of AGN emission in the $K$ band. Indeed, the $\mathrm{CO}_{\text {spec-obs }}$ values of a large fraction of the observed Seyfert 2 galaxies in Figure $2 a$ are substantially smaller than the typical values observed in star-forming or elliptical (=spheroidal) galaxies $\left(\mathrm{CO}_{\text {spec }}>0.15\right.$; Goldader et al. 1997a, 1997b; Ivanov et al. 2000). This is likely to be caused by dilution of the $\mathrm{CO}$ absorption feature by the AGN's featureless continuum emission.

In the $L$ band, the emission from stars is much reduced when compared to that in the $K$ band, whereas hot $(\sim 1000 \mathrm{~K})$ dust heated by an AGN has strong emission at both $K$ and $L$. Thus, the relative contribution from the AGN to the nuclear spectra increases substantially from $K$ to $L$ (Alonso-Herrero et al. 1996, 2001). In fact, the typical $K-L$ color of an AGN is usually larger (redder) than a normal stellar population 
TABLE 2

Near-Infrared Properties of the Observed Seyfert 2 Galaxies

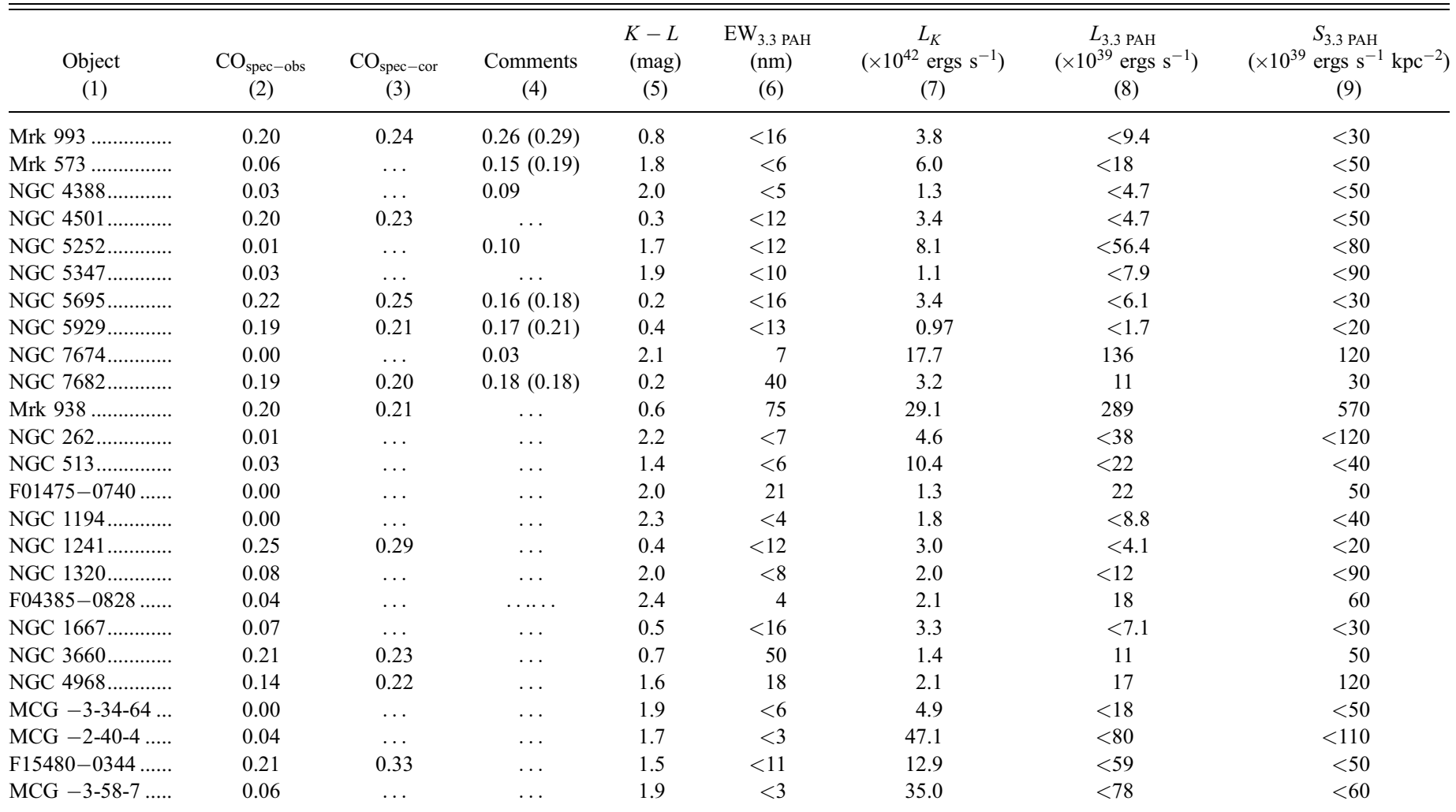

Notes.-Col. (1): object; col. (2): observed spectroscopic CO index; col. (3): corrected spectroscopic CO index; col. (4): observed (corrected) spectroscopic CO index taken from Ivanov et al. (2000); col. (5): nuclear $K-L$ color in magnitude, measured with our slit spectroscopy; col. (6): rest-frame equivalent width of the $3.3 \mu \mathrm{m}$ PAH emission feature in nm, taken from Imanishi (2003); col. (7): $K$-band stellar luminosity, defined as $\lambda L_{\lambda}$ at $2.2 \mu \mathrm{m}$; col. (8): observed 3.3 $\mu \mathrm{m}$ PAH luminosity, taken from Imanishi (2003); col. (9): surface brightness of the $3.3 \mu \mathrm{m}$ PAH emission feature.

(Willner et al. 1984; Alonso-Herrero et al. 2003). The $K-$ $L$ colors can be used to estimate the AGN contribution to the nuclear $K$-band spectra. The nuclear $K-L$ colors, derived from our slit spectra, are summarized in column (5) of Table 2. Figure $3 a$ compares the nuclear $K-L$ colors with the $\mathrm{CO}_{\text {spec-obs }}$ values. The $K-L$ color and $\mathrm{CO}_{\text {spec-obs }}$ are anticorrelated in such a way that objects with redder $K-L$ colors tend to show smaller $\mathrm{CO}_{\text {spec-obs. }}$. The probability that a corre- lation is not present is found to be $0.01 \%$ (Isobe et al. 1986). Since the observed $K-L$ colors should become redder, when compared to those of a normal stellar population, for an increasing contribution from the AGN, the anticorrelation in Figure $3 a$ suggests that the smaller $\mathrm{CO}_{\text {spec-obs }}$ values in objects with redder $K-L$ colors are caused by the AGN contribution. This clearly demonstrates that to investigate the stellar emission from the $\mathrm{CO}$ absorption features in more
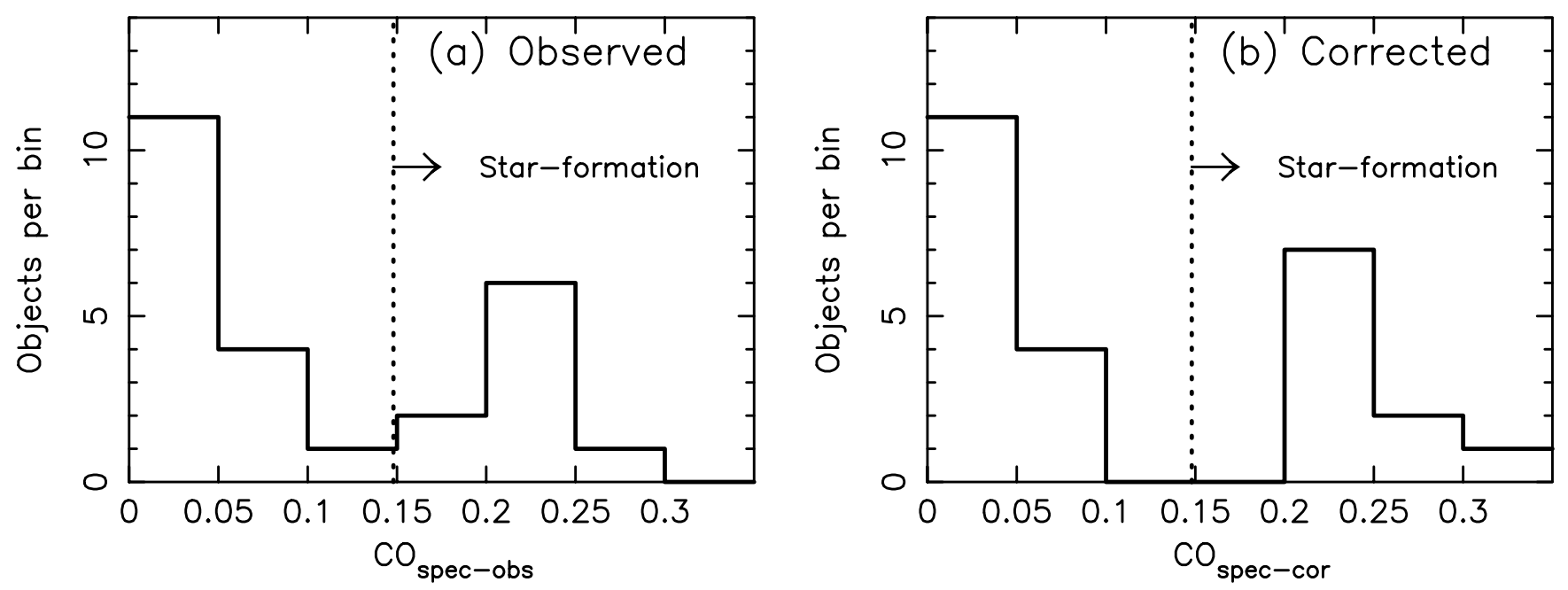

FIG. 2. - (a) Histogram of the observed $\mathrm{CO}$ index $\left(\mathrm{CO}_{\text {spec-obs }}\right)$. (b) Histogram of the corrected $\mathrm{CO}$ index $\left(\mathrm{CO}_{\text {spec-cor }}\right)$. For sources with observed $\mathrm{CO}$ indices $<0.10$, no AGN correction was attempted. Star-forming or elliptical galaxies typically show $\mathrm{CO}_{\text {spec }}>0.15$ (Goldader et al. 1997a, 1997b; Ivanov et al. 2000). 

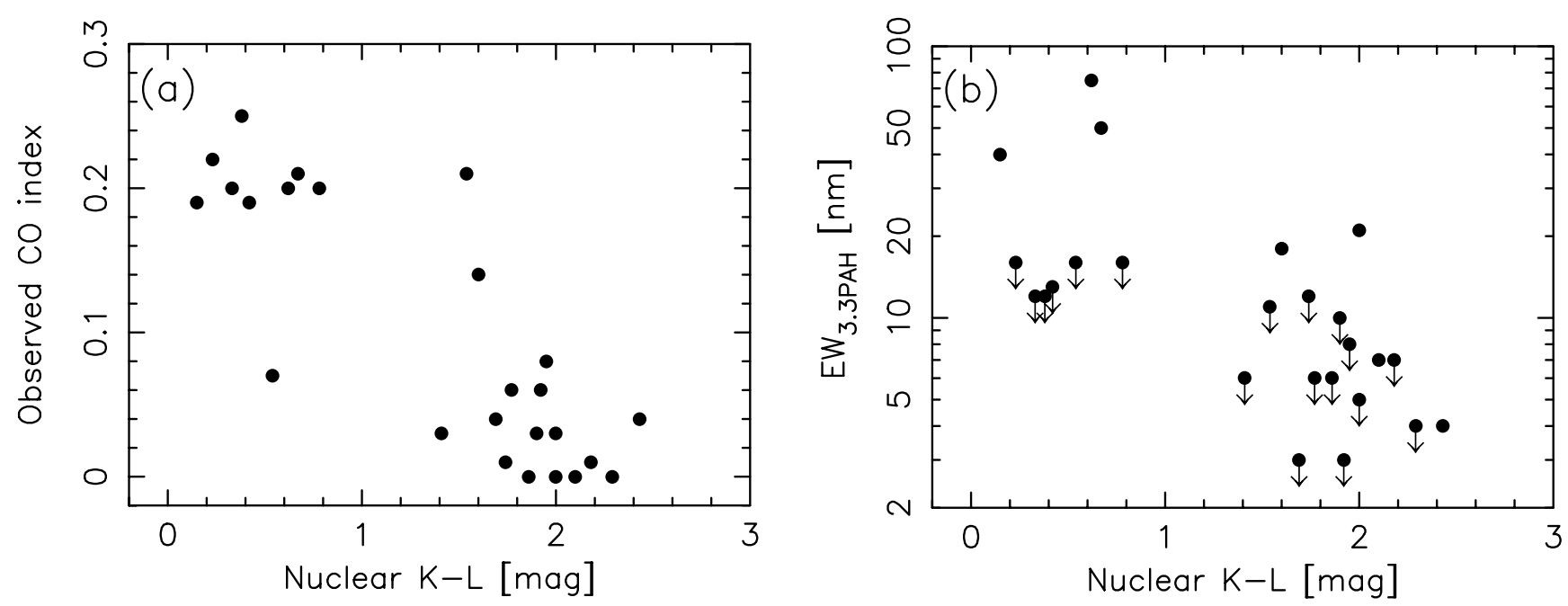

FIG. 3. - (a) Nuclear $K-L$ magnitude (abscissa) and observed spectroscopic CO index (ordinate). The uncertainty of the $K-L$ color is $<0.1$ mag. (b) The ordinate represents the rest-frame equivalent width of the $3.3 \mu \mathrm{m}$ PAH emission feature. Its uncertainty is at a level of $<30 \%$ for the PAH-detected sources.

detail, a correction for the AGN dilution is necessary. To make this correction quantitatively, we make use of the restframe equivalent width of the $3.3 \mu \mathrm{m}$ PAH emission feature $\left(\mathrm{EW}_{3.3 \mathrm{PAH}}\right)$. The $\mathrm{EW}_{3.3 \mathrm{PAH}}$ values are summarized in column (6) of Table 2.

Hot dust heated by an AGN produces a featureless $L$-band continuum, while star-forming galaxies usually show strong $3.3 \mu \mathrm{m}$ PAH emission with $\mathrm{EW}_{3.3 \mathrm{PAH}} \sim 100 \mathrm{~nm}$ (Imanishi \& Dudley 2000). Since the equivalent width is by definition robust to dust extinction, we assume that pure star formation shows $\mathrm{EW}_{3.3 \mathrm{PAH}}=100 \mathrm{~nm}$, while a pure AGN shows $\mathrm{EW}_{3.3 \mathrm{PAH}}=0 \mathrm{~nm}$. For the three Seyfert 2 galaxies with

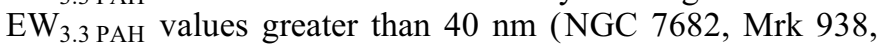
and NGC 3660; Imanishi 2003), a significant contribution from star formation to the $L$-band fluxes must be present. For these sources, we estimate the fractional stellar contribution at

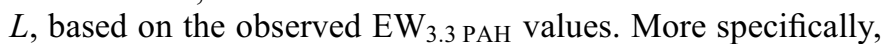
if the observed $\mathrm{EW}_{3.3 \mathrm{PAH}}$ value is $70 \mathrm{~nm}$, we regard that $70 \%$ of the observed $L$-band flux comes from star formation, and the remaining $30 \%$ originates in AGN emission. We then extract $L$-band fluxes originated in AGN activity from the observed $L$-band flux levels. For the Seyfert 2 galaxies with very small $\mathrm{EW}_{3.3 \text { РАH }}$ of $\$ 20 \mathrm{~nm}$ ( 22 sources except NGC 7682, Mrk 938, and NGC 3660), we regard the observed $L$-band fluxes as dominated by AGN-powered emission (Imanishi 2003). Namely, the observed $L$-band fluxes are taken as AGNoriginated $L$-band fluxes.

To derive the AGN fraction in the $K$-band spectra from the estimated AGN-powered $L$-band flux levels, we assume that the intrinsic AGN colors in all Seyfert 2 galaxies are $K-L=$ 2.79 , as was done by Ivanov et al. (2000). Alonso-Herrero et al. (2003) have shown that the infrared AGN spectral energy distributions in Seyfert 2 galaxies are fitted with $f_{\nu} \propto \nu^{-3}$, which provides $K-L \sim 2.5-2.6$. Thus, the adopted assumption of $K-L=2.79$ is reasonably justified from the observational point of view. After subtracting this AGN emission from the observed $K$-band spectra, we can infer the stellar $K$-band emission and thereby obtain corrected $\mathrm{CO}$ indices $\left(\mathrm{CO}_{\text {spec-cor }}\right)$. The derived stellar $K$-band luminosities are summarized in column (7) of Table 2. Regarding the corrected $\mathrm{CO}$ index $\left(\mathrm{CO}_{\text {spec-cor }}\right)$, if the AGN contribution to the observed $K$-band flux is estimated to be $60 \%$, then the $\mathrm{CO}$ absorption optical depth increases by $2.5[=1 /(1-0.6)]$, and the spectroscopic CO index increases accordingly. Thus, when the observed CO index is very small, say $\mathrm{CO}_{\text {spec-obs }}<0.1$, because of dilution from AGN emission at $K$, a small uncertainty in the continuum determination can result in a large ambiguity in the corrected $\mathrm{CO}$ index. There are 10 sources with $\mathrm{CO}_{\text {spec-obs }}>0.1$. We regard the $\mathrm{CO}$ absorption as clearly detected in these 10 sources and estimate $\mathrm{CO}_{\text {spec-cor }}$ only for them, in order for the correction process to work reasonably. The $\mathrm{CO}_{\text {spec-cor }}$ values of the 10 sources are summarized in column (3) of Table 2, and the histogram of the $\mathrm{CO}$ indices corrected in this way is plotted in Figure $2 b$. Although the AGN correction certainly increases the $\mathrm{CO}$ indices, the change of their overall distribution is little. Thus, possible ambiguities of this AGN correction do not seriously affect our main conclusions.

This method to correct the $\mathrm{CO}$ indices for AGN emission using our own slit spectra has important advantages over some other methods, such as that based on nuclear $K$ - and $L$-band photometric data. First, since the $K$ - and $L$-band spectra were taken simultaneously under the same observing conditions and probe the same regions in the observed Seyfert 2 galaxies, there is little ambiguity on the flux intercalibration. Second, the compact nuclear $L$-band emission observed in Seyfert galaxies is usually assumed to be dominated by AGN emission (Ivanov et al. 2000). Although this is true for the majority of Seyfert 2 galaxies (Imanishi 2002, 2003), there are some exceptions. From our $L$-band slit spectra, we can clearly pick out these exceptions on the basis of the $\mathrm{EW}_{3.3 \mathrm{PAH}}$ values and take into account the stellar contamination to the nuclear $L$-band fluxes.

However, there is one caution for this correction process. The PAH emission observed in star-forming regions primarily comes from photodissociation regions located between $\mathrm{H}$ II regions and molecular gas, and not from the $\mathrm{H}$ II regions themselves (Sellgren 1981). If the star-forming regions have such a high radiation density that their emitting volumes are predominantly occupied by $\mathrm{H}$ II regions with virtually no photodissociation regions, then no $3.3 \mu \mathrm{m}$ PAH emission is expected. Although this is the case for the nuclear star cluster in the dwarf galaxy NGC 5253 (Alonso-Herrero et al. 2004), this kind of star-forming regions is probably a minor exception. Figure $3 b$ compares the nuclear $K-L$ color with $\mathrm{EW}_{3.3 \mathrm{PAH}}$ for our sample of Seyfert 2 galaxies. Using a statistical test by Isobe et al. (1986), the probability that a correlation is not 


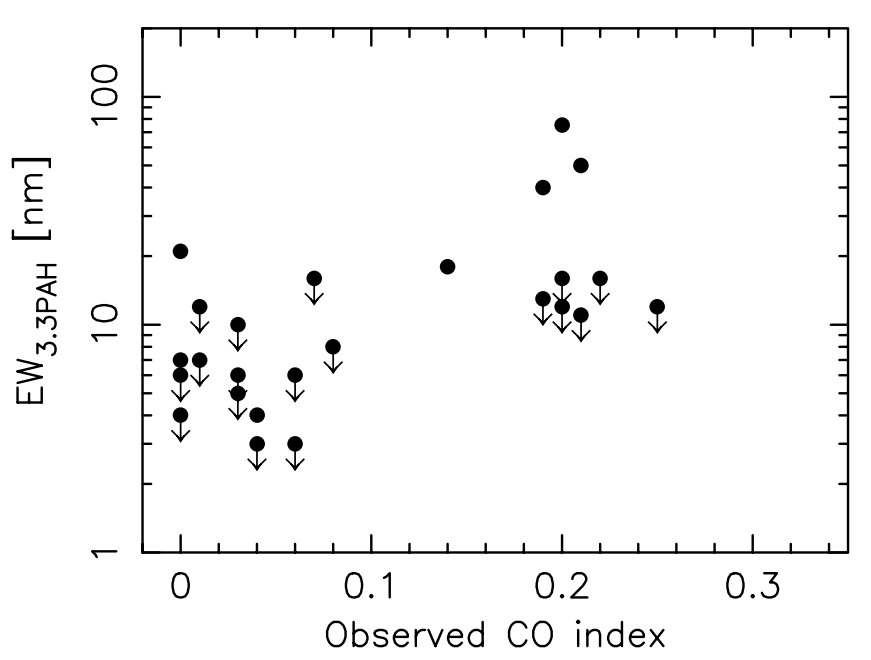

FIG. 4.-Observed spectroscopic CO index (abscissa) and rest-frame equivalent width of the $3.3 \mu \mathrm{m}$ PAH emission feature (ordinate).

present is found to be $30 \%$, suggesting an anticorrelation between the $K-L$ and $\mathrm{EW}_{3.3 \mathrm{PAH}}$, as expected from the assumption that the featureless $L$-band continuum originates in the AGN. If emission from pure $\mathrm{H}$ II regions were contributing significantly to the nuclear $L$-band featureless continuum in many Seyfert 2 galaxies, then the estimated AGN contribution to the nuclear $K$-band flux would be overestimated. In this case, the corrected $\mathrm{CO}$ indices would be smaller than the above estimates.

In Figure $2 b 40 \%(=10 / 25)$ of the observed Seyfert 2 galaxies have corrected $\mathrm{CO}$ indices $\left(\mathrm{CO}_{\mathrm{spec}-\mathrm{cor}}\right)$ that are as large as those of star-forming or elliptical (=spheroidal) galaxies $\left(\mathrm{CO}_{\text {spec }}>0.15\right.$; Goldader et al. 1997a, 1997b; Ivanov et al. 2000). Since very young star formation, with less than a few million years in age, should show very small $\mathrm{CO}_{\text {spec }}$ values (Leitherer et al. 1999), such star formation is missed in this $K$-band CO absorption method. Furthermore, when the AGN dilution is very severe in the $K$ band, the $\mathrm{CO}$ absorption features are very weak and so the correction of the observed spectroscopic $\mathrm{CO}$ indices for the AGN dilution is not possible. In fact, for NGC 7674 and F01475-0740, although ongoing nuclear star formation is indicated from the detected $3.3 \mu \mathrm{m}$ PAH emission feature, no clear $\mathrm{CO}$ absorption features are detected. Thus, this fraction $(=40 \%)$ should be taken as a lower limit of Seyfert 2 nuclei with stellar emission.

\subsection{The Nature of the Stellar Emission Detected in the K Band}

The stellar signatures detected in the nuclei of Seyfert 2 galaxies can be due to nuclear starbursts in the AGN torus and/or old bulge (=spheroid) stars. Since both starbursts and old bulge stars show $\mathrm{CO}_{\text {spec }} \gtrsim 0.15$, these two populations are not easily distinguishable on the basis of the $\mathrm{CO}_{\text {spec }}$ values alone (Oliva et al. 1995). To further understand the nature of the detected stellar emission in the $K$ band, we combine the $K$-band data with the $L$-band spectra (Imanishi 2003). Figure 4 compares the observed $\mathrm{CO}$ index with $\mathrm{EW}_{3.3 \mathrm{PAH}}$ (shown in col. [6] of Table 2). They are weakly correlated.

Imanishi (2003) implicitly assumed that the detected $3.3 \mu \mathrm{m}$ PAH emission originates in the putative nuclear starbursts in the AGN torus. Here we test this assumption in a quantitative manner on the basis of the $3.3 \mu \mathrm{m}$ PAH emission luminosity measured in our apertures, because starbursts and old bulge stars are distinguishable in terms of emission surface

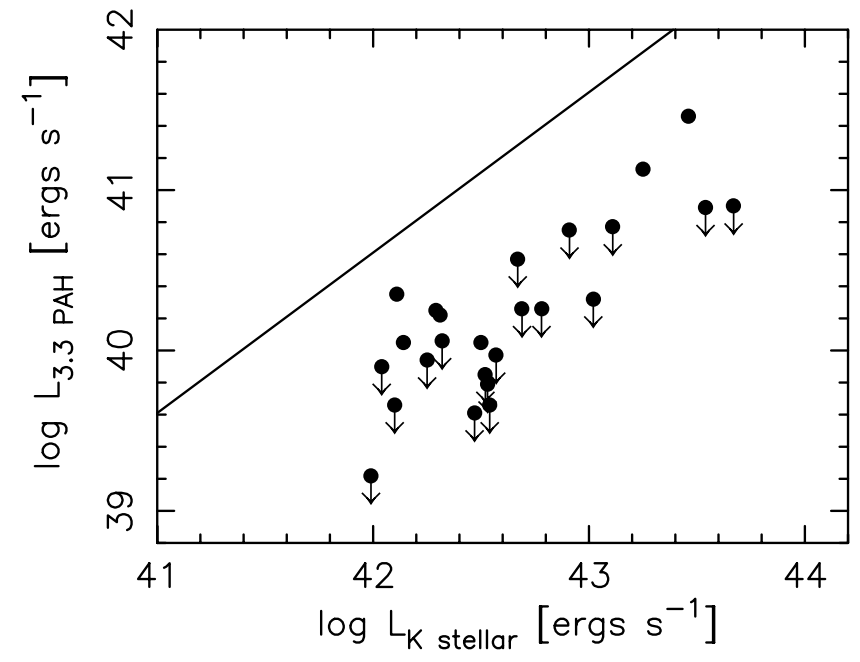

FIG. 5.-Observed nuclear stellar $K$-band luminosity, defined as $\lambda L_{\lambda}$ at $2.2 \mu \mathrm{m}$ (abscissa) and nuclear $3.3 \mu \mathrm{m}$ PAH luminosity estimated from our slit spectra (ordinate). The errors of the PAH luminosities for the PAH-detected sources are at a level of $<30 \%$. The solid line indicates the correlation for infrared-luminous starburst galaxies (see text).

brightness. Heckman (2001) estimated a star formation rate per unit area of $10^{-1} M_{\odot} \mathrm{yr}^{-1} \mathrm{kpc}^{-2}$ as the lower limit beyond which the superwind activity, a characteristic property of starbursts, can occur. This limit corresponds to an infrared surface brightness of $S_{\mathrm{IR}} \sim 2 \times 10^{42} \mathrm{ergs} \mathrm{s}^{-1} \mathrm{kpc}^{-2}$ (Kennicutt 1998), or a $3.3 \mu \mathrm{m}$ PAH emission surface brightness of $S_{3.3 \text { PAH }} \sim 2 \times 10^{39} \mathrm{ergs} \mathrm{s}^{-1} \mathrm{kpc}^{-2}$ (Imanishi 2002). Among the 25 Seyfert 2 galaxies studied in this paper, the $3.3 \mu \mathrm{m} \mathrm{PAH}$ emission was detected by Imanishi (2003) in the seven sources; NGC 7674, NGC 7682, Mrk 938, F01475-0740, F043850828, NGC 3660, and NGC 4968. The surface brightnesses of the $3.3 \mu \mathrm{m}$ PAH emission are summarized in column (9) of Table 2. For all the PAH-detected sources, the observed surface brightnesses significantly exceed the threshold required for starbursts. Therefore, for the PAH-detected Seyfert 2 nuclei, we can conclude that the detected $3.3 \mu \mathrm{m}$ PAH emission must come from a nuclear starburst and cannot be of old stellar origin.

Figure 5 compares the $K$-band stellar luminosity with the $3.3 \mu \mathrm{m}$ PAH emission luminosity from the Seyfert 2 nuclei measured from our slit spectra. In infrared-luminous starburst galaxies, the $K$-band to infrared luminosity ratios are found to be $L_{K} / L_{\mathrm{IR}} \sim 10^{-1.6}$ (Goldader et al. 1997a). The $3.3 \mu \mathrm{m}$ PAH to infrared luminosity ratios in starbursts are estimated to be $L_{3 . \mathrm{PAH}} / L_{\mathrm{IR}} \sim 10^{-3}$ (Mouri et al. 1990; Satyapal et al. 1995; Imanishi 2002). Thus, the $3.3 \mu \mathrm{m} \mathrm{PAH}$ to $K$-band luminosity ratios are expected to be $L_{3.3 \mathrm{PAH}} / L_{K} \sim 10^{-1.4}$ if both luminosities trace starbursts. This value is shown as a solid line in Figure 5. The observed ratios in the Seyfert 2 galaxies in our sample are smaller, by a large factor, than the value expected for starbursts. If the starbursts are very young, with ages of less than a few million years, then $L_{K} / L_{\mathrm{IR}}$ decreases (Goldader et al. 1997a), and thus the discrepancy between the observed and expected $L_{3.3 \mathrm{PAH}} / L_{K}$ ratios is even larger.

Figure 5 suggests that in the nuclei of Seyfert 2 galaxies, compared to starbursts, $L_{3.3 \mathrm{PAH}}$ is underluminous or $L_{K}$ is overluminous. One possibility for the $L_{3.3}$ PAH underluminosity is the destruction of PAH-emitting material by energetic radiation from the central AGN (Voit 1992). However, since the putative nuclear starbursts are expected to occur at the outer 

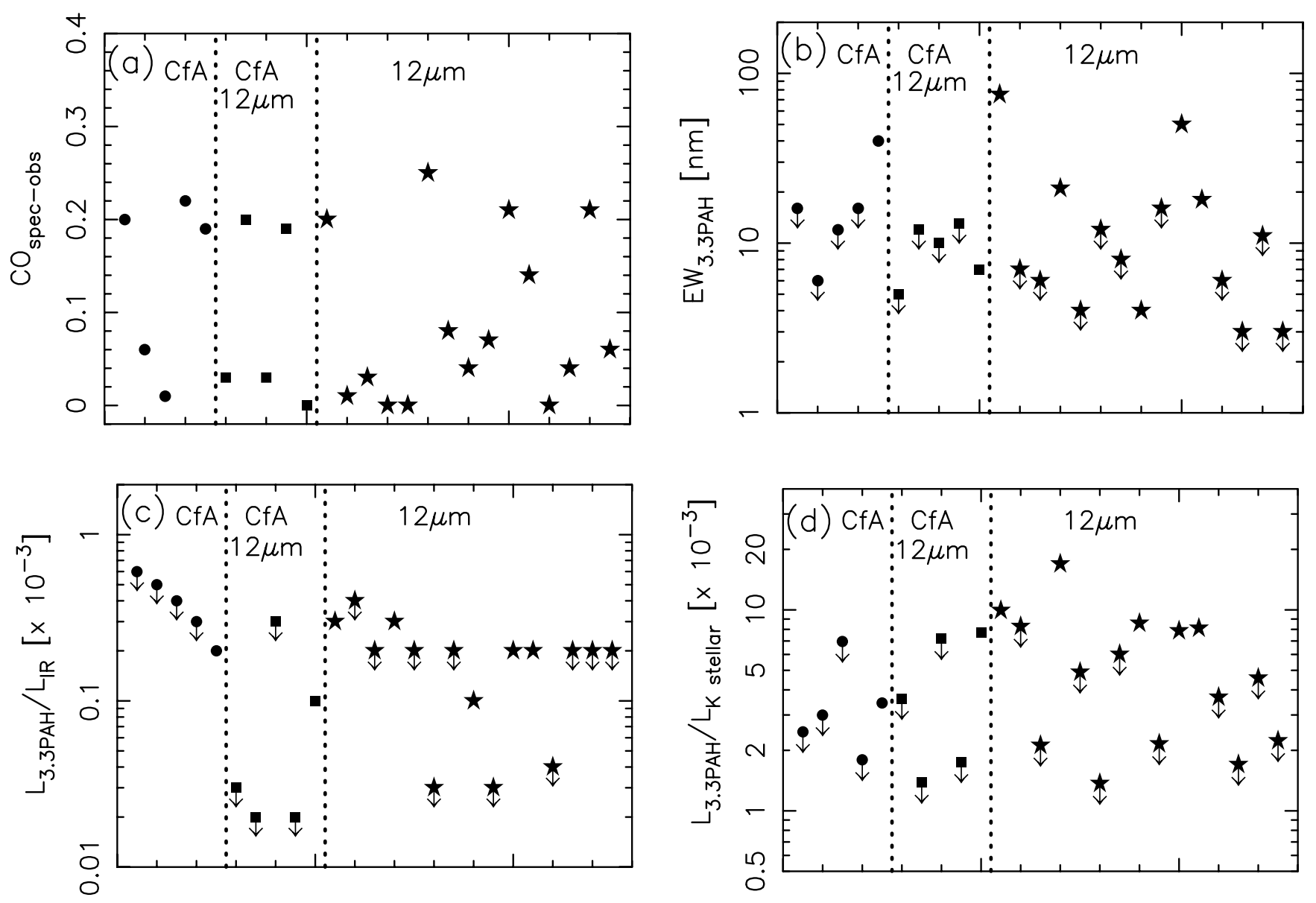

FIg. 6.-(a) Distribution of the observed spectroscopic CO index. Circles, CfA Seyfert 2 galaxies; stars, $12 \mu \mathrm{m}$ Seyfert 2 galaxies. Five sources listed in both samples are plotted as squares. (b) The rest-frame equivalent width of the $3.3 \mu \mathrm{m}$ PAH emission feature. (c) The $3.3 \mu \mathrm{m}$ PAH to infrared luminosity ratio, taken from Imanishi (2003). (d) The $3.3 \mu \mathrm{m}$ PAH to stellar $K$-band luminosity ratio.

part of the obscuring torus where the AGN radiation is significantly attenuated (Heckman et al. 1997; Imanishi 2003), this destruction is unlikely to be severe. Aside from the PAH destruction by the AGN radiation, the PAH emission may be suppressed if the stellar radiation density is so high that the volume fraction of photodissociation regions relative to $\mathrm{H}$ II regions is substantially smaller than typical starbursts. If the PAH destruction in the nuclear starbursts in the AGN tori were more severe than typical starburst galaxies, the actual infrared luminosity of the nuclear starbursts would be substantially larger than the estimate based on the assumption of $L_{3.3 \mathrm{PAH}} / L_{\mathrm{IR}} \sim 10^{-3}$ (Imanishi 2003). However, we regard this possibility as unlikely, because for a few selected Seyfert 2 galaxies for which the luminosities of the nuclear starbursts were estimated from both the UV and $3.3 \mu \mathrm{m}$ PAH emission (assuming $L_{3.3 \mathrm{PAH}} / L_{\mathrm{IR}} \sim 10^{-3}$ ), the estimated nuclear starburst luminosities agree quantitatively well (Imanishi 2002).

We suggest that our $K$ - and $L$-band results are more naturally explained by a scenario in which the $3.3 \mu \mathrm{m}$ PAH emission originates in the spatially unresolved (smaller than subarcsecond) nuclear starbursts in the AGN torus, whereas the signatures of stellar emission detected in the $K$-band spectra are produced mainly by old bulge stars. In fact, the nuclear $L$-band emission of Seyfert 2 galaxies is dominated by spatially unresolved emission rather than the bulge component, whereas the extended stellar components often contribute predominantly to the nuclear $K$-band emission (Alonso-Herrero et al. 1996, 1998, 2001). Old bulge stars can contribute significantly to the nuclear $K$-band fluxes but not to the PAH emission, owing to the paucity of PAH-exciting far-UV photons in bulges compared to starbursts. This scenario explains the small $L_{\mathrm{PAH}} / L_{K}$ ratios observed in Figure 5. In this scenario, the CO absorption strength is primarily due to old bulge stars rather than nuclear starbursts, and thus it may not be a useful tool to detect the putative nuclear starbursts in the AGN torus. This scenario is consistent with the conclusion by Ivanov et al. (2000).

\subsection{Do the CfA and $12 \mu \mathrm{m}$ Seyfert 2 Galaxies Contain Different Nuclear Stellar Properties?}

To improve the statistics, so far we have discussed nearinfrared emission properties by combining both the CfA and $12 \mu \mathrm{m}$ Seyfert 2 galaxies together. However, since selection criteria of these samples are different, a separate discussion of Seyfert 2 galaxies in each sample is of interest.

Figure 6 plots the distribution of $\mathrm{CO}_{\text {spec-obs, }} \mathrm{EW}_{3.3 \mathrm{PAH}}$, $L_{3.3 \mathrm{PAH}} / L_{\mathrm{IR}}$, and $L_{3.3 \mathrm{PAH}} / L_{K}$ stellar. In all the plots, no systematic differences are found between Seyfert 2 galaxies in the CfA and $12 \mu \mathrm{m}$ samples. Thus, the main arguments in this paper are taken to be applicable to Seyfert 2 stellar populations in general. In particular, the $L_{3.3 \mathrm{PAH}} / L_{\mathrm{IR}}$ ratios in Figure $6 c$ and $L_{3.3 \mathrm{PAH}} / L_{K}$ stellar in Figure $6 d$ trace the relative contribution from the nuclear starbursts to the infrared and nuclear stellar $K$-band luminosity, respectively (Imanishi 2003). There was a possibility that the $12 \mu \mathrm{m}$ Seyfert 2 galaxies may be biased to those with powerful nuclear starbursts 
(Ho \& Ulvestad 2001), because the starbursts can be strong emitters at $12 \mu \mathrm{m}$. The absence of systematic differences in Figures $6 c$ and $6 d$ suggests that no such strong bias is present in the $12 \mu \mathrm{m}$ Seyfert 2 sample.

\section{CONCLUSION}

We have presented results from a near-infrared $K$-band slit spectroscopic survey of 25 Seyfert 2 galaxies in the CfA and $12 \mu \mathrm{m}$ samples. These data have been combined with results from the $3.3 \mu \mathrm{m}$ PAH emission survey of Imanishi (2003). The $\mathrm{CO}$ absorption features at $\lambda_{\text {rest }}=2.3-2.4 \mu \mathrm{m}$ have been used to investigate the properties of the stellar populations in the nuclei of Seyfert 2 galaxies. We have found that the observed values of the $\mathrm{CO}$ indices in a large fraction of Seyfert 2 nuclei are affected by dilution produced by the featureless continuum associated with the AGN, because Seyfert 2 nuclei with redder $K-L$ colors tend to show shallower CO absorption features. For sources with modestly large observed CO indices $(>0.1)$, we have corrected the $\mathrm{CO}$ indices for AGN dilution. This correction has made a small change for the overall distribution of the $\mathrm{CO}$ indices in our sample and we have estimated that $\sim 40 \%(=10 / 25)$ of Seyfert 2 nuclei have CO indices similar to those of star-forming or elliptical (=spheroidal) galaxies. This is only a lower limit of Seyfert 2 nuclei with stellar emission because young star formation shows no $\mathrm{CO}$ absorption features and because in Seyfert 2 nuclei where the $K$-band light is dominated by AGN emission, the correction of the observed $\mathrm{CO}$ indices for AGN dilution is not possible.
A comparison between the nuclear $3.3 \mu \mathrm{m}$ PAH to stellar $K$-band luminosity ratio in the nuclei of Seyfert 2 galaxies and observations for infrared luminous starburst galaxies implies that the $\mathrm{CO}$ absorption features detected in the nuclear $K$-band spectra of Seyfert 2 galaxies are due mainly to old bulge (=spheroid) stars rather than the putative nuclear starbursts in the AGN torus, and thus that the putative starbursts are difficult to detect with $K$-band spectra over the scales probed by our data $(250 \mathrm{pc}$ to $1 \mathrm{kpc})$. We found no clear difference in the properties of the $2.3 \mu \mathrm{m} \mathrm{CO}$ absorption and $3.3 \mu \mathrm{m}$ PAH emission features between the CfA and $12 \mu \mathrm{m}$ Seyfert 2 samples.

We are grateful to J. Rayner, S. Bus, P. Sears, and W. Golisch for their support during our IRTF SpeX observing runs, to K. Wada for useful discussions, and to the referee for valuable comments. M. I. is supported by a Grant-in-Aid for Scientific Research (16740117). A. A. H. acknowledges support from the Spanish Programa Nacional de Astronomía y Astrofísica under grant AYA2002-01055. Some part of the data analysis was made using a computer system operated by the Astronomical Data Analysis Center (ADAC) and the Subaru Telescope of the National Astronomical Observatory, Japan. This research has made use of the SIMBAD database, operated at CDS, Strasbourg, France, and of the NASA/IPAC Extragalactic Database (NED), which is operated by the Jet Propulsion Laboratory, California Institute of Technology, under contract with the National Aeronautics and Space Administration.
Alonso-Herrero, A., Quillen, A. C., Rieke, G. H., Ivanov, V., \& Efstathiou, A. 2003, AJ, 126, 81

Alonso-Herrero, A., Quillen, A. C., Simpson, C., Efstathiou, A. \& Ward, M. J. 2001, AJ, 121, 1369

Alonso-Herrero, A., Simpson, C., Ward, M. J., \& Wilson, A. S. 1998, ApJ, 495, 196

Alonso-Herrero, A., Takagi, T., Baker, A. J., Rieke, G. H., Rieke, M. J., Imanishi, M., \& Scoville, N. Z. 2004, ApJ, 612, 222

Alonso-Herrero, A., Ward, M. J., \& Kotilainen, J. K. 1996, MNRAS, 278, 902 Antonucci, R. 1993, ARA\&A, 31, 473

Arnaud, K. A., Gilmore, G., \& Cameron, A. C. 1989, MNRAS, 237, 495

Coziol, R., Doyon, R., \& Demers, S. 2001, MNRAS, 325, 1081

Doyon, R., Joseph, R. D., \& Wright, G. S. 1994, ApJ, 421, 101

Fabian, A. C., Barcons, X., Almaini, O., \& Iwasawa, K. 1998, MNRAS, 297, L11

Goldader, J. D., Joseph, R. D., Doyon, R., \& Sanders, D. B. 1997a, ApJ, 474, 104

. 1997b, ApJS, 108, 449

Gonzalez Delgado, R. M., Heckman, T., Leitherer, C., Meurer, G., Krolik, J., Wilson, A. S., Kinney, A., \& Koratkar, A. 1998, ApJ, 505, 174

Heckman, T. M. 2001, in ASP Conf. Ser. 240, Gas and Galaxy Evolution, ed. J. E. Hibbard, M. Rupen, \& J. H. van Gorkomastro (San Francisco: ASP), 345

Heckman, T. M., Gonzalez Delgado, R., Leitherer, C., Meurer, G. R., Krolik, J.,

Wilson, A. S., Koratkar, A., \& Kinney, A. 1997, ApJ, 482, 114

Ho, L. C., \& Ulvestad, J. S. 2001, ApJS, 133, 77

Huchra, J., \& Burg, R. 1992, ApJ, 393, 90

Imanishi, M. 2002, ApJ, 569, 44 2003, ApJ, 599, 918

Imanishi, M., \& Dudley, C. C. 2000, ApJ, 545, 701
Isobe, T., Feigelson, E. D., \& Nelson, P. I. 1986, ApJ, 306, 490

Ivanov, V. D., Rieke, G. H., Groppi, C. E., Alonso-Herrero, A., Rieke, M. J., \& Engelbracht, C. W. 2000, ApJ, 545, 190

Kennicutt, R. C., Jr. 1998, ARA\&A, 36, 189

Kohno, K., Matsushita, S., Vila-Vilaro, B., Okumura, S. K., Shibatsuka, T., Okiura, M., Ishizuki, S., \& Kawabe, R. 2002, in ASP Conf. Ser. 249, The Central kpc of Starbursts and AGN, ed. J. H. Knapen, J. E., Beckman, I. Shlosman, \& T. J. Mahoney (San Francisco: ASP), 672

Le Floc'h, E., Mirabel, I. F., Laurent, O., Charmandaris, V., Gallais, P., Sauvage, M., Vigroux, L., \& Cesarsky, C. 2001, A\&A, 367, 487 Leitherer, C., et al. 1999, ApJS, 123, 3

Mouri, H., Kawara, K., Taniguchi, Y., \& Nishida, M. 1990, ApJ, 356, L39

Oliva, E., Origlia, L., Kotilainen, J. K., \& Moorwood, A. F. M. 1995, A\&A, 301,55

Oliva, E., Origlia, L., Maiolino, R., \& Moorwood, A. F. M. 1999, A\&A, 350, 9

Rayner, J. T., Toomey, D. W., Onaka, P. M., Denault, A. J., Stahlberger, W. E.,

Vacca, W. D., Cushing, M. C., \& Wang, S. 2003, PASP, 115, 362

Rhee, J. H., \& Larkin, J. E. 2000, ApJ, 538, 98

Rush, B., Malkan, M. A., \& Spinoglio, L. 1993, ApJS, 89, 1

Satyapal, S., et al. 1995, ApJ, 448, 611

Sellgren, K. 1981, ApJ, 245, 138

Tokunaga, A. T. 2000, in Allen's Astrophysical Quantities, ed. A. N. Cox (4th ed; Berlin: Springer), 143

Vanzi, L., Alonso-Herrero, A., \& Rieke, G. H. 1998, ApJ, 504, 93

Veilleux, S., Goodrich, R. W., \& Hill, G. J. 1997, ApJ, 477, 631

Voit, G. M. 1992, MNRAS, 258, 841

Wada, K., \& Norman, C. A. 2002, ApJ, 566, L21

Willner, S. P., Ward, M., Longmore, A., Lawrence, A., Fabbiano, G., \& Elvis, M., 1984, PASP, 96, 143 